\title{
Electrochemical Sensor based on Polyethyleneimine-AuNPs- Anthraquinone-2-carboxylic acid Nanocomposite for Cysteine Detection
}

\author{
Manman Li ${ }^{1}$, Zhuangzhuang Guo ${ }^{1}$, Huaixia Yang ${ }^{1}$, Yanju Liu ${ }^{1, *}$, Yan Tong ${ }^{1}$, Jinming Kong ${ }^{2, *}$ \\ ${ }^{1}$ Pharmacy College, Henan University of Chinese Medicine, Zhengzhou 450008, P. R. China. \\ ${ }^{2}$ School of Environmental and Biological Engineering, Nanjing University of Science and \\ Technology, Nanjing 210094, P. R. China. \\ *E-mail: liuyanju886@ hactcm.edu.cn, j.kong@njust.edu.cn
}

doi: $10.20964 / 2019.01 .72$

Received: 30 September 2018 / Accepted: 6 November 2018 / Published: 30 November 2018

\begin{abstract}
A novel electrochemical cysteine (Cys) sensing strategy based on the modification of glassy carbon electrodes with nanocomposite is presented in this paper. This proposed sensor exhibits excellent sensitivity and high selectivity toward Cys with a detection limit of $1.873 \times 10^{-6} \mathrm{M}$. Features of the modified electrode were characterized by ultraviolet spectrophotometry (UV), contact angle (CA), transmission electron microscopy (TEM) and cyclic voltammetry (CV) methods. The reaction mechanism was explored by density functional theory, results suggested that the interaction forces between anthraquinone-2-carboxylic acid (AQCA) and Cys were hydrogen bond. Furthermore, this sensor successfully detected Cys in human serum samples with satisfactory recovery ranges, indicating a promising application in biological samples analysis and clinical diagnosis.
\end{abstract}

Keywords: Gold nanoparticle; Polyethyleneimine; Anthraquinone-2-carboxylic acid; Cysteine; electrochemical sensing

\section{FULL TEXT}

(C) 2019 The Authors. Published by ESG (www.electrochemsci.org). This article is an open access article distributed under the terms and conditions of the Creative Commons Attribution license (http://creativecommons.org/licenses/by/4.0/). 